\title{
Correlación entre pensamiento táctico y el desarrollo de las habilidades técnicas y físicas de jugadores de fútbol infantil Correlation between tactical thought and the development of the technical and physical skills of child soccer players
}

\author{
Saúl Ignacio Vega O rozco, Fernando Bernal Reyes, 0 mar Iván Gavotto N ogales, Héctor Manuel Sarabia Sainz, \\ Mario Alberto Horta Gim \\ Universidad de Sonora(M éxico)
}

\begin{abstract}
Resumen. El presente trabajo pretende evidenciar lacorrelación entreel nivel de pensamiento táctico delosjugadores de fútbol infantil de nivel iniciación en lamejorade las habilidadestécnicas y físicas. M etodología: participaron 26 jugadores defútbol infantil de ocho y nueve años con dos grupos: un grupo con desigualdad numéricay otro con igualdad, ambos con 13 participantes. A los dos gruposse les aplicó una batería de pruebasfísicasy técnicas, así como el test del conocimiento táctico ofensivo en el fútbol (TCTO F). Losgrupos estuvieron expuestos aun programadeentrenamiento de 16 sesiones. Resultados: Losjugadores pertenecientesal nivel muy bajo, solo mostraron cambiossignificativos negativos en la correlación flexibilidad-fuerza en piernas y pase-conducción del grupo con igualdad numérica. Losjugadores de nivel bajo mostrar on cambios significativos positivos en las correlaciones de flexibilidad-fuerza en piernas, flexibilidad-cabeceo y cabeceo-fuerza en piernas. En cambio, los participantes denivel regular (el más elevado en nuestrainvestigación) produjeron correlaciones significativas positivas entre golpeo de pierna no dominante-flexibilidad y estemismo gol peo con laresistenciaaeróbicaen el grupo con desigualdad numérica. Por su parte, el grupo con igualdad numérica presentó diferenciassignificativasentre lafuerza-flexibilidad, velocidad-flexibilidad, pase-flexibilidad, pase-fuerzaen piernas, pase-velocidad, pase-resistencia, pase-cabeceo, gol peo-regate, gol peo-conducción y gol peo con piernano dominantecabeceo. Conclusión: existe una correlación positiva entre el nivel de pensamiento táctico ofensivo en el fútbol con el desarrollo de las habilidades técnicasy físicas, es decir, mientras mayor seaestenivel, mayores cambios significativosseproducirán en sus habilidades.
\end{abstract}

Palabras clave: fútbol infantil, pensamiento táctico, técnica, capacidadesfísicas.

\begin{abstract}
This work aimsto evidence the correlation between the level of tactical thinking of the starting level children'sfootball playersin improving technical and physical skills. M ethodology: 26 eight- and nine-year-old children's football players participated with two groups: one group with numerical inequality and one with equality, both with 13 participants. The two groups were given a battery of physical and technical tests, as well as the test of tactical offensive knowledge in football (TCTOF). The groups were exposed to a 16-session training program. Results: Playersat the very low level only showed changessignificant negativein the correlation the flexibility and strength of the legs and pass and running of the ball in the group with numerical equality. Low-level players showed significant positive changes in correlations of flexibility and strength in legs, flexibility and heading, and heading and strength in legs. In contrast, regular-level participants (the highest in our research) produced significant positive correlations between shooting the ball with thenon-dominant leg and flexibility and this same shooting the ball with the non-dominant leg with the aerobic resistance in the group with numerical equality. For its part, the group with numerical equality presented significant differences between strength and flexibility, speed and flexibility, pass the ball and flexibility, pass the ball and force in legs, pass the ball and speed, pass the ball and resistance, pass and heading the ball, shoot the ball with dominant leg and dribbling, shoot the ball with dominant leg and running with the ball, and heading the ball with shoot the ball non-dominant leg. Conclusion: there is a positive correlation between the level of tactical offensive thinking in football with the development of technical and physical skills, the higher this level, the greater significant changes in your skills.
\end{abstract}

Keywords: child soccer. tactical thinking, technical skills. physical abilities.

\section{Introducción}

En la actualidad, en el fútbol iniciación se han ido desarrollando muchas corrientes y enfoques para el desarrollo de las habilidades propias del deporte, ya que, por sus características de deporte colectivo de dos equipos, siempre existe la confrontación entre los mismos (Corcho, González \& Arrojo, 2016). Durante estas actividades, los jugadores deben pensar y actuar casi al mismo tiempo, por el poco tiempo que tienen en cada

Fecha recepción: 11-11-20. Fecha de aceptación: 26-11-20

Saúl Ignacio Vega O rozco

saul.vega@unison.mx situación, por lo que se le llama también pensamiento operativo o pensamiento táctico (Cañizares, 2008). Las acciones tácticas responden a intenciones previas, incluyen aspectos técnicos, físicos, cognitivos y emocionales; uno de los aspectos más importantes es la toma de decisiones (Corcho et al., 2016), por ello, la importancia de un desarrollo integral en estos factores en pro de la mejora de la toma de decisiones en las acciones globales del juego.

En el fútbol, se ha comprobado que la superioridad numérica que se obtiene tras una expulsión aumenta el rendimiento colectivo (Aguilar, Chirosa, Martín \& Chirosa, 2012). En este sentido, se han realizado inves- 
tigaciones en balonmano y waterpolo con la implementación de programas con desigualdad numérica e igual dad numérica, las cuales señalan que la desigualdad proporciona cambios significativos en la toma de decisiones y en la ejecución técnica (García, Argudo \&Alonso, 2015).

El objetivo del estudio es analizar la correlación entre el nivel del pensamiento táctico y el desarrollo de las capacidades técnicas y físicas en dos programas de entrenamiento. La hipótesis de nuestro estudio es que mientras mayor sea el nivel de pensamiento táctico de los jugadores, mayores serán los desarrollos en sus ca pacidades técnicas y físicas.

\section{M etodología}

Se basó en el enfoque cuantitativo de la investiga ción científica, donde se usó el diseño experimental, de característicade estudio Iongitudinal, con dos grupos de estudio para identificar si existe diferencia entre los resultados pre-test y pos-test. Posteriormente, se correlacionó el nivel de pensamiento táctico con las variables analizadas.

\section{Participantes}

En el estudio, se analizaron a 26 jugadores de fútbol infantil de género masculino, pertenecientes al equipo de la categoría ocho-nueve años del Centro Educativo CDIAIFaEs de la ciudad de Hermosillo, Sonora, MéxiCO.

Para la distribución homogénea de los participantes en el estudio, se utilizó un método alterno para intentar hacer inicialmente equivalentes los grupos, la cual fue el emparejamiento o técnica de apareo (matching), la cual consiste en medir una o algunas variables (se midieron todas en el pre-test) para después ordenarlas de las puntuaciones más altas a las más bajas y después asignar de manera equitativa en los grupos (ver tabla 1), así mismo se menciona que entre más variables se utilicen para aparear grupos, el procedimiento se vuelve más complejo (Hernández, Fernández \& Baptista, 2014).

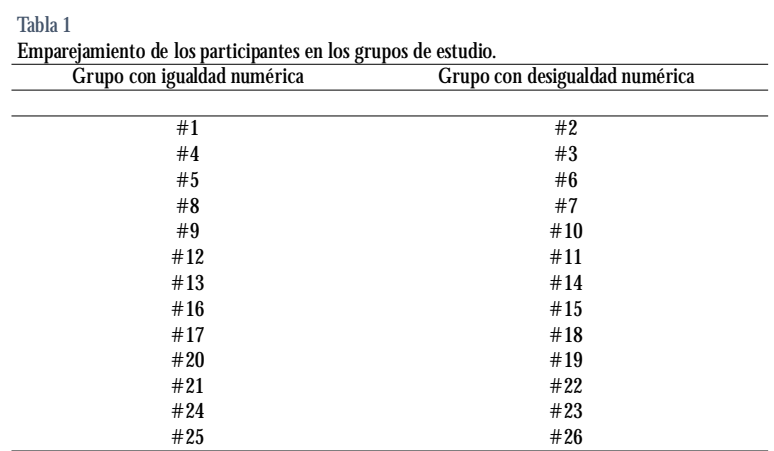

\section{Grupo con igualdad numérica}

Estuvo conformado por 13 jugadores varoniles con una edad media de $8.3 \pm 0.6$ años y con un grado de experienciade 3.2 años de práctica de fútbol iniciación. Este grupo fue sometido a un programa de entrenamiento de agrupación igualitaria, es decir, los objetivos de las sesiones de entrenamiento se agrupaban en uno versus uno, dos versus dos, tres versus tres, cuatro versus cuatro, cinco versus cinco y seis versus seis. Los ejercicios mayormente utilizados fueron juegos de espacios reducidos y los juegos globales.

\section{Grupo con desigualdad numérica}

Estuvo conformado por 13 integrantes quienes presentaban una edad media de $8.0 \pm 0.7$ años y contaban con 2.7 años de experiencia entrenando fútbol de etapa iniciación. Este fue sometido a un programa con desigualdad numérica en la agrupación dentro de las tareas de entrenamiento, donde se presentaban situaciones dos versus uno, tres versus dos, cuatro versus tres, cinco versus cuatro, seis ver sus cinco y siete versus seis. Aquí también los juegos de espacios reducidos y los juegos globales fueron los más utilizados.

Ambos programas tenían los mismos objetivos generales y específicos por sesión, lo que los diferenciaba uno de otro, era la forma de agrupación dentro de las tareas de entrenamiento. Las sesiones tenían una periodicidad de dos días por semana con una duración de 105 minutos por sesión; la cual se dividía en tres partes: calentamiento, parte principal y vuelta a la calma.

\section{Criterios de inclusión}

Los padres de los alumnos debían asistir a una junta informativa donde se les darían los detalles de la investigación y firmarían el consentimiento informado para que los alumnos participaran en dicha investigación, además, debían estar debidamente inscritos en el centro educativo CDI AIFaEs, pertenecer al equipo de fútbol de la categoría de fútbol ocho y nueve años de la rama varonil y asistir con regularidad a los días de entrenamiento donde se impartiría el programa de entrenamiento.

\section{Criterios de exclusión}

Presentar algún impedimento físico, discapacidad motriz o coronaria que impidiera realizar las pruebas físicas y técnicas. así como la ejecución del programa de entrenamiento; presentar alguna lesión o patología que impida el desarrollo óptimo de los participantes. Tampoco podían pertenecer a una selección de fútbol infan- 
til que represente en torneos de manera local, municipal o nacional, por no considerarse futbolistas de nivel iniciación.

\section{Instrumentos}

En la parte técnica se utilizaron los test de la batería «the soccer star» de: conducción en línea recta, de golpeo de balón con ambos pies, de regate y el de cabeceo utilizada por Bernal (2015) y el «Soccer Passing Test» utilizada por M ulazimoglu (2014). Para la parte física: el salto de longitud(García, 2001), el test de sprint en línea recta 20metros (Calleja-González, Los Arcos, M ejuto, Casamichan, San Roman-Q uintana \& Yanci 2015), el test de Course Navette (Leger, Mercier,Gadoury \& Lambert, 1988) para estimar el V02 máximo y el test de conocimiento táctico ofensivo en el fútbol «TCTOF» (Serra-Olivares \& García López, 2016) para identificar el nivel del pensamiento táctico.

La otra variable para correlacionar fue el nivel del pensamiento táctico obtenido al finalizar el programa, donde por medio del test de pensamiento táctico ofensivo en el fútbol, se establecieron los porcentajes y categorías para asignar niveles (tabla 2). Cabe señalar que en la investigación solo se obtuvieron de los niveles regular, bajo y muy bajo.

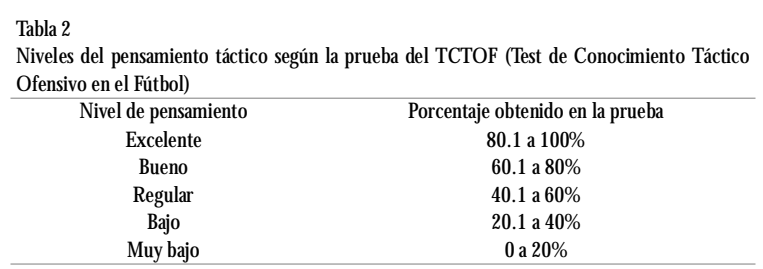

\section{Análisis estadístico}

Se aplicó la prueba de normalidad Shapiro-W ilk, después a los datos con distribución normal se les aplicó una «T de Student» y para los no paramétricos se recurrió aW ilcoxon, estas para determinar los efectos pretest pos-test. Posteriormente, se aplicó un análisis de correlación de Pearson para determinar la influencia del nivel del pensamiento táctico y el desarrollo de las variables medidas. Se trabajó con un nivel de confianza del $95 \%$ y un nivel de significancia de $p<0,05$.

\section{Resultados}

Para establecer las correlaciones, se observaron los resultados en el pre-test y post-test de ambos grupos, tal como lo ilustra la tabla 3, donde se apreciaron cambios significativos en la mayoría de las habilidades y ca pacidades, a excepción del pase $(p=.005)$, cabeceo $(p=.001)$ y flexibilidad $(p=.035)$ que solo se mejoraron en el grupo con desigualdad numérica, el cual fue sometido al programa de desigualdad numérica en las ta reas de entrenamiento.

Tabla 3.

Resultados de las pruebas físicas y técnicas (pre y post) categorizados por grupos.

Grupo con igualdad numérica Grupo con desigualdad numérica \begin{tabular}{cccccccc}
\multicolumn{2}{c}{ Pre-test } & Post-test & Sig & Pre-test & Post-test & Sig. \\
\hline Media & Ds & Media & Ds & & Media & Ds Media Ds & \\
\hline
\end{tabular} Regate(seg) $\quad 33.07 \pm 7.28 \quad 28.30 \pm 7.80 .003 * 33.65 \pm 5.6 \quad 27.45 \pm 5.57 .000^{*}$ Conducción (seg) $\quad 8.87 \quad \pm 2.08 \quad 7.25 \pm 2.05 .000 * \quad 8.51 \pm 1.16 \quad 6.95 \pm .86 .000^{*}$

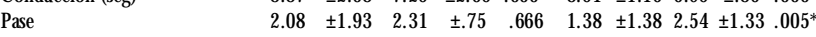
$\begin{array}{lllllllllll}\text { Cabeceo } & 1.46 & \pm .96 & 1.92 & \pm .76 & .190 & .85 & \pm .98 & 1.85 & \pm .98 & .001^{*}\end{array}$ Golpeo piernadominante $\quad 2.23 \quad \pm 2.42 \quad 4.62 \pm 1.66 .005^{*} \quad 2.31 \pm 2.13 \quad 5.92 \pm 2.06 .001 *$ Golpeo con pierna no dominante $1.15 \quad \pm 2.30 \quad 3.23 \quad \pm 2.00 \quad .000^{*} \quad 1.69 \pm 1.84 \quad 3.38 \pm 1.26 .011^{*}$ Flexibilidad (cm) $\quad 14.4 \quad \pm 7.1 \quad 13.9 \pm 6.4 \quad 632 \quad 18.3 \pm 5.0816 .54 \pm 5.62 .035^{*}$

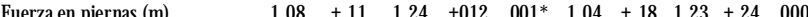
$\begin{array}{llllllllll}\text { Fuerza en piernas }(m) & 1.08 & \pm .11 & 1.24 & \pm 012 & .001 * & 1.04 & \pm .18 & 1.23 & \pm .24\end{array}$ Velocidad

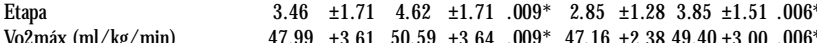
Vo2máx $(\mathrm{m} / / \mathrm{kg} / \mathrm{min}) \quad 47.99 \pm 3.61 \quad 50.59 \pm 3.64 .009 * 47.16 \pm 2.3849 .40 \pm 3.00 .006^{*}$

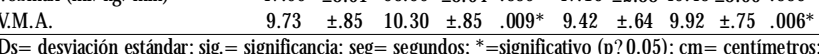
$\mathrm{m}=\mathrm{metros}, \mathrm{ml} / \mathrm{kg} / \mathrm{min}=$ mililitros/ kilogramos/ minuto y v. m. a. = velocidad máxima al canzada

En primer lugar, se analizaron las correlaciones de los jugadores de nivel muy bajo, donde solamente se apreciaron dos correlaciones negativas significativas en el grupo con desigualdad numérica, una entre la fuerza en piernas y la flexibilidad (-.952) que en la medida de que crece la fuerza en piernas disminuye la flexibilidad y de manera inversa cuando la flexibilidad aumenta la fuerza disminuye. Así mismo, encontramos una correlación negativa entre el pase y la conducción del balón en línea recta (-.982) de manera inversa y proporcional, esto se aprecia en la tabla 4. El grupo con igualdad numérica no mostró correlaciones positivas o negativas.

\section{Tabla 4}

Correlaciones de Pearson del nivel del pensamiento táctico "M uy Bajo" con las diferencias obtenidas en las evaluaciones físicas y técnicas. Categorizadas por grupo.

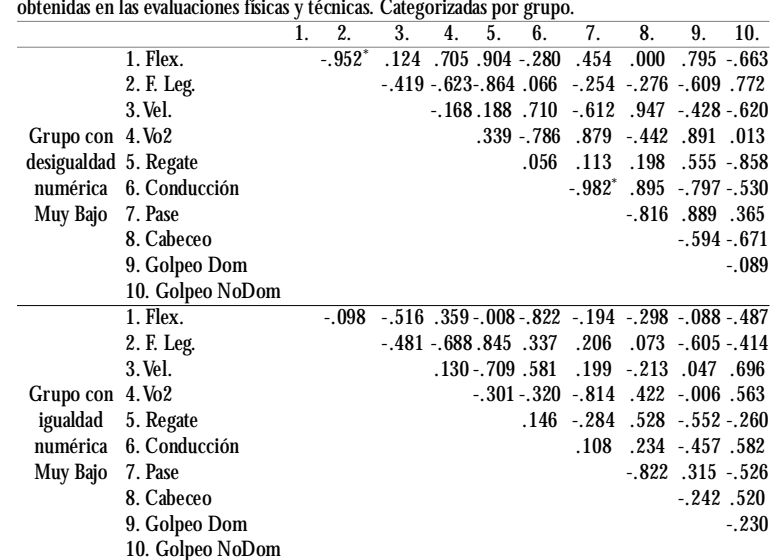

Flex. = Flexibilidad; F. Leg. = Fuerza en piernas; Vel. = Velocidad; V02= Volumen máximo de 0 xigeno; Reg $=$ Regate; $C$ ond $=$ Conducción; $C a b .=C a b e c e 0 ; G$ olpeo Dom. $=$ Golpeo con pierna dominante; Golpeo $\mathrm{NoD}$ om $=$ Golpeo con pierna no dominante $\mathrm{y}^{*}=$ la correlación es significativa en el nivel 0.05 (bilateral).

Los jugadores con nivel bajo de pensamiento táctico ofensivo del grupo con desigualdad numérica presentaron una correlación positiva entre la habilidad del rega te y el golpeo del balón con la pierna dominante (.922) es decir, a medida que una variable mejora la otra se ve 
directamente afectada, por lo que cambios favorables en el regate favorecen el desarrollo del golpeo del ba lón con la pierna dominante y viceversa. También se encontró una correlación negativa en el pase y el golpeo del balón con la pierna menos dominante (-.872). En este mismo sentido, pero en el grupo con igualdad numérica, la flexibilidad se correlacionó de manera positiva con la fuerza en piernas $(.846)$ y con el cabeceo (.841) la cual provocaba alteraciones de manera lineal en estos dos apartados; así mismo, estas dos variables se correlacionaron de manera positiva entre ellas (.869) creando un triángulo entre estos tres factores en este grupo, tal como se expresa en la tabla 5.

Tabla 5

Correlaciones de Pear son del nivel del pensamiento táctico "Bajo" con las diferencias obtenidas en las evaluaciones físicas y técnicas. Categorizadas por grupo.

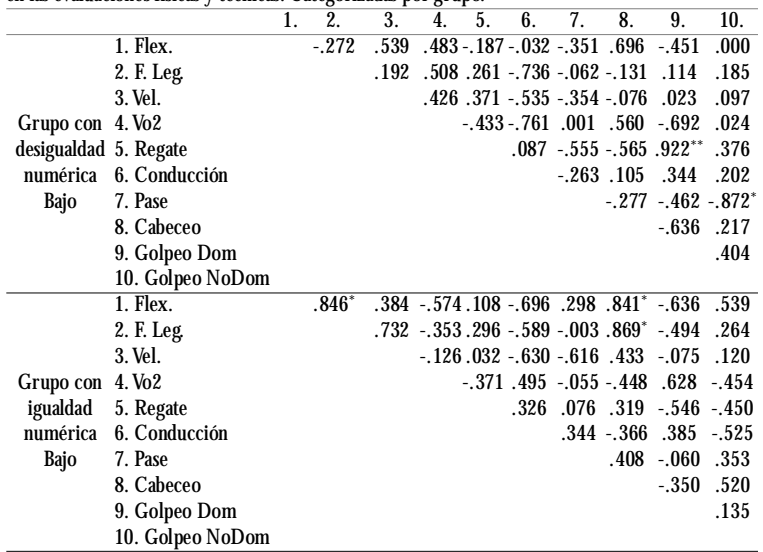

Flex. $=$ Flexibilidad; F. Leg. = Fuerza en piernas; Vel. $=$ Velocidad; Vo2= Volumen máximo de 0 xigeno; Reg $=$ Regate; Cond $=$ Conducción $;$ Cab. $=$ Cabeceo; Golpeo Dom. $=$ Golpeo con pierna dominante; Golpeo NoDom $=$ Golpeo con pierna no dominante $y^{*}=$ la correlación es significativa en el nivel 0.05 (bilateral).

Finalmente, los jugadores que poseían un nivel de pensamiento regular (el más elevado de nuestra investigación) mostraron la mayor cantidad de correlaciones en ambos grupos. En el grupo con desigual dad numérica, se mostraron correlaciones entre la flexibilidad y el

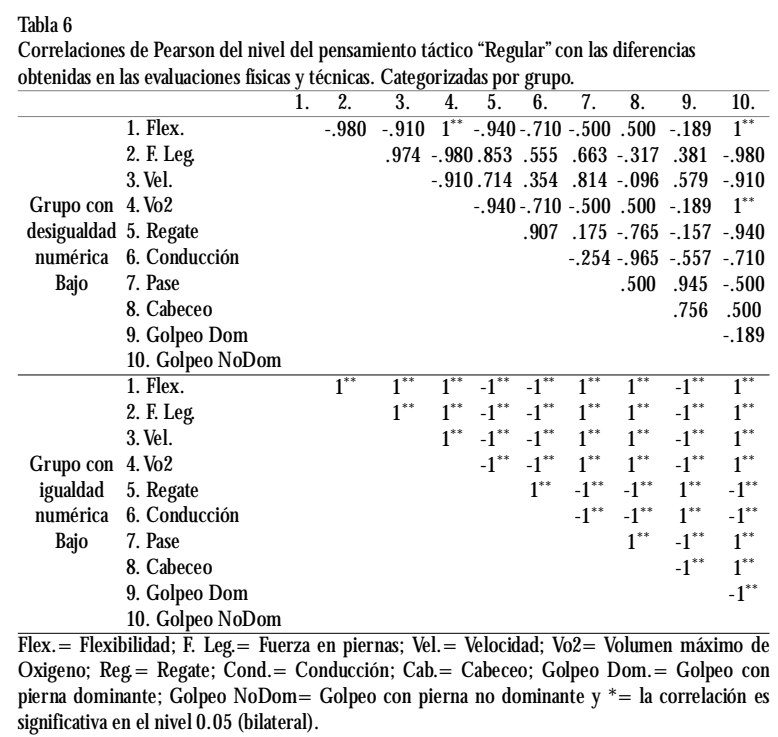

$\overline{\text { Flex }}$ = Flexibilidad; F. Leg. $=$ Fuerza en piernas; Vel. $=$ Velocidad; Vo2 = Volumen máximo de Oxigeno; Reg $=$ Regate; $C$ ond $=$ Conducción; $\mathrm{Cab} .=\mathrm{Cabeceo} ; \mathrm{Golpeo} \mathrm{Dom} .=\mathrm{Golpeo}$ con pierna dominante; Gol peo NoDom $=$ Golpeo con pierna no dominante $y^{*}=$ la correlación es significativa en el nivel 0.05 (bilateral).

volumen de oxígeno máximo (1.000); entre la flexibilidad y el golpeo del balón con pierna no dominante (1.000), así como la interacción entre el golpeo del balón con pierna no dominante y el volumen de oxígeno máximo (1.000). Por su parte, en el grupo con igualdad numérica se presentaron muchas correlaciones positivas y negativas, tal como se ilustra en la tabla 6 . Donde se aprecian mayores cor relaciones positivas es en el fundamento del pase, el cual en los resultados de las evaluaciones fue el que sufrió mejores diferencias significa tivas durante la investigación, lo que se relaciona positivamente con la flexibilidad, la fuerza en piernas, la velocidad, el volumen máximo de oxígeno, el cabeceo y el golpeo con la pierna no dominante (1.000).

\section{Discusión}

Algunas investigaciones muestran la relación directa entre las habilidades técnico-deportivas con las capacidades físicas, y mencionan que mientras más desarroIladas se muestren las capacidades de agilidad, coordinación y velocidad, mayor rendimiento técnico deportivo tendrán (M iranda, Manuel, Jos \& Zamora 2015).

0 tro aspecto muy importe en el ámbito del pensamiento táctico, es el grado de pericia de los jugadores, y esto lo comprueba Sánchez, De laVega-Marcos, RuízBarquín \& Del Valle (2012), en su estudio, donde menciona que los jugadores con un grado de pericia más elevado poseen mejores resultados en aspectos de táctica individual como en la táctica colectiva de equipo. Así mismo, estos autores sugieren la utilización de otras pruebas de selección de la muestra, enfocadas al grado de inteligencia, como la prueba de matices progresivas de Raven, con el fin de conseguir muestras más homogéneas, ya que esta capacidad está íntimamente ligada a latomade decisiones. Atendiendo a este punto, Gimeno, Buceta \& Peréz-Llantada (2007), menciona la relevancia del trabajo psicológico dirigido a desarrollar una confianza adecuada en sus propias posibilidades técnicas, en el trabajo que realizan en los entrenamientos y en sus expectativas en competiciones deportivas.

Gómez (2014) reafirma la dinámica de este programa de intervención, ya que mencionan que todos los elementos estructurales del fútbol: reglas de juego, espacio de juego, tiempo, relación entre participantes, el balón y la portería son susceptibles de manipulación de cara a los entrenamientos contextualizados. Por ende, en futuras líneas de investigación se pudieran estudiar estas variables para evidenciar las mejoras por dicha experimentación. De igual forma, como lo expresan 
Campos (2015) en la actualidad es más asiduo la integración de contenidos técnicos, tácticos y condicionales dentro de las tareas de entrenamiento de forma globalizada.

En la investigación realizada por Reytor, Hernández \& Lorenzo (2017), concluyen que los indicadores de mayor incidencia en el pensamiento táctico operativo se centran en la selección de la respuesta motora más ideal de acuerdo con la situación. Así mismo, algunas investigaciones, como la de Salinero, González-M illan, Ruíz-Vicente, Abian, García-Aparicio, Rodríguez-Ca brero \& Cruz (2013), realizaron eval uaciones técnicas para correlacionarlas con la edad y la velocidad de toma de decisiones, donde claramente se ven diferencias significativas entre los más chicos (10-11 años) con los más grandes (16-17 años), pero esto también se debe a que las evaluaciones se realizaron de manera analítica y por separado, por lo que sería conveniente, realizar este tipo de comparaciones en situaciones globalizadas o situaciones reales del contexto práctico (partidos), mismo caso de este sistema de evaluación de las capacidades físicas, técnicas y tácticas, las cuales fueron evalua das por separado y de manera aisladas.

0 tro dato interesante y con miras en la misma línea de investigación, es el expuesto por Guijarro-Romero, M ayorga-Vega \& Viciana (2017), donde compararon el nivel de habilidad táctica entre varones y mujeres, en el cual concluyen que los varones poseen valores significativamente mayores que las mujeres y recomiendan el desarrollo de tareas y estrategias didácticas específicas para igualar estas diferencias de género.

\section{Conclusión}

Existe una correlación entre el nivel de pensamiento táctico ofensivo en el fútbol y el mejoramiento de las habilidades técnicas y físicas de los jugadores de fútbol infantil, es decir, entre mayor es el nivel, mayores son los cambios significativos en las diferencias pre y post test de las capacidades físicas y habilidades técnicas de los jugadores de fútbol infantil.

Se recomienda la implementación de un programa específico para la mejora del pensamiento táctico ofensivo y la utilización de elementos que desarrollen estas capacidades en los jugadores de fútbol de nivel inicia ción, así mismo, como este instrumento evalúa la parte delas decisiones y acciones (procedimentales) en la parte ofensiva, sería interesante ver los aspectos que conforman el pensamiento de juego en la fase defensiva en estudios posteriores.

\section{Referencias}

Aguilar, J., Chirosa, L., M artín, I., \& C hirosa, I. (2012). Influenciadel número de jugadores/ as en latomade decisiones y el rendimiento en la enseñanzadel balonmano. E-balonmano. com:Revista deCiencias del Deporte, 8(3), 253-263. Recuperado de https:// dialnet.unirioja.es/ servlet/ articulo?codigo $=4375218$

Bernal, F. (2015). Incidencia dediferentesmetodologíasdeenseñanza defútbol en niños de 8 a 11 años, sobrel os aspectostécnicosy la diversión, en la ciudad deH ermosillo, Sonora, Mexico.TesisD octoral. Universidad de León.

Calleja_González, J., LosArcos, A., M ejuto, G., Casamichana, D., San RomanQ uintana, J., \&Yanci, J. (2015). Reproducibilidad detest de aceleración y cambio dedirección en fútbol. RICYDE: Revisa Internacional deCienciasdel Deporte, 11(40), 104-115. https: / doi.org/ 10.5232/ ricyde

CamposV ázquez, M. A. (2015). M onitorización de respuestasfísicasy fisiológicasal entrenamiento y la competición en fútbol. TesisD octoral. U niversidad Pablo de Olavide.

Cañizares, M. (2008). La psicología en la actividad física. Su apl icación en la educación física, el deporte, la recreación y la rehabilitación. La Habana: Editorial D eportes.

Corcho Busto, H. M., González Padrón, C. Á., \& Arrojo Sureda, A. (2016). 0 ptimización del pensamiento táctico apartir dejuegossimplificadosdefútbol en iniciación deportiva. SPORTTK:Revista Euroamericana de Cienciasdel De porte, 6(1), 81-90. . Recuperado dehttps: / / revistas. um.es/ sportk/ article/ view/ 280451

García, J. (2001). Programa Eurofit. MECD.

GarcíaM arín, P., Argudo Iturriaga, F., \&Alonso Roque, J. (2015). Laacción de juego en desigualdad numéricapor periodos en waterpolo. Retos nuevastendenciasen educaciónfísica, deportey recreación, 27, 14-18. Recuperado de: Redalyc. La acción de juego en desigualdad numéricapor periodosen waterpolo

Gimeno, M ., Buceta, J. M ., \& Pérez-Llantada, M . C. (2007). Influenciadelasvariablespsicológicasen el deportede competición: evaluación medianteel cuestionar io Características psicológicascon el rendimiento deportivo. Psicothema, 19(0214-9915), 667-672. https:// www. redalyc. org/ articulo. oa?d $=72719420$

GómezP., P. (2014). Preparación Física para el fútbol. M eixco:Trillas.

Guijarro-Romero, S. , M ayorga-Vega, D., \&Viciana, J. (2017). Influence of gender on the tactical skill and motivational aspects in invasion sports in physical education. Cultura, Ciencia y D eporte, 14 (41), 93-105. https:/ / doi. org/ $10.12800 / \mathrm{ccd} . v 14 i 41.1269$

Hernández Sampieri, R., Fernández Collado, C., \& Baptista Lucio, P. (2014). Metodología dela investigación. M éxico: McGraw-Hill.

Leger, L., Mercier, D., Gadoury, C., \& Lambert,J. (1988).Themultistage 20 metre ShuttleR un test for aerobic fitness. J SportsSci 6 (2) 93-101 1988. Journal of sportssciences, 6, 93-101. https:/ / doi.org/ 10.1080/ 02640418808729800

Miranda, F., M anuel, H., J os, D., \& Zamora, A. (2015). Análisis de correlación entrecapacidadesfísicasy rendimiento técnico-deportivo, en niños principiantes en fútbol. Ef deportes, 209,6. ). Recuperado de https:// dialnet. unirioja.es/ servlet/ articulo?codigo $=6110341$

Mulazimoglu, 0. (2014). An investigation of the effect of fatigue on passing accuracy in soccer players. International Journal of Academic Research, 6(2), 259- 267. https:/ / doi.org/ 10.7813/ 2075-4124.2014/ 6-2/ a.37

Reytor Pérez, P., Hernández Hernández, M., \& Lorenzo Bertheau, E. (2017). El entrenamiento mental del equipo 1 racategoríade fútbol degranmadurante el campeonato nacional deacenso de latemporada (original). O limpia: Publicación científica del a facultad decultura física dela Universidad de Granma, 14(42), 24-34. Recuperado de https:/ / dialnet. unirioja.es/ servlet/ articulo?codigo $=6210631$

Salinero, J. J., González-Millán, C., Ruíz-Vicente, D., A biánVicén, J., GarcíaAparicio,A., Rodríguez-Cabrero, M., \& Cruz, A. (2013). Valoración dela condición Físicay técnicaen fútbolistasjóvenes. Revisa Internacional de M edicina y Cienciasdela Actividad Fisca y del D eporte, 13(50), 401-418. Recuperado dehttp:/ / cdeporte.rediris. es/ revista/ revista50/ artval oracion354. htm

Sánchez, R., de laVega-M arcos, R., Ruíz-Barquín, R., \& D elValle, S. (2012). Análisisy evaluación de los niveles de toma de concienciay comprensión táctica en fútbol por medio de un tablero de juego. SPORT TK-Revista EuroAmericana de Ciencias del Deporte, 1(1 SE-), 33-38. https:/ / doi. org/ $10.6018 / 185481$

Serra O livares, J. \& G García López, L. M . (2016). Diseño y validación del test de conocimiento táctico ofensivo en fútbol (TCTO F) / Design andValidation of theSoccerTactical KnowledgeTest (STKT). Revisa Internacional deM edicina y CienciasdelaActividad Físca y del Deporte, 63 (2016), 521-536. https:/ / doi.org/ 10.15366/ rimcafd2016.63.008 